\title{
Aplicação de reguladores vegetais em uva apirena 'Centennial Seedless'
}

\author{
Growth regulators applied on seedless grape 'Centennial Seedless'
}

\author{
Willian Rodrigues Macedo ${ }^{\mathrm{I}}$ Maurilo Monteiro Terra' ${ }^{\mathrm{II}}$ Marco Antonio Tecchio ${ }^{\mathrm{III}}$ \\ Erasmo José Paioli Pires ${ }^{\text {II }}$ Gisele Machado Fernandes ${ }^{\text {IV }}$ \\ Larissa VillarI Mara Fernandes Moura'II
}

\section{RESUMO}

O trabalho objetivou avaliar o efeito de doses crescentes do forchlorfenuron associado ou não ao ácido giberélico sobre componentes físico-químicos da uva 'Centennial Seedless' para consumo in natura. Os tratamentos testados foram ácido giberélico ( $\left.A G_{3}\right)\left(0\right.$ e $\left.5 \mathrm{mg} \mathrm{L}^{-1}\right)$ associado ao forchlorfenuron $\left(0,2,4,6,8\right.$ e $\left.10 \mathrm{mg} \mathrm{L}^{-1}\right)$. As variáveis analisadas foram a massa, o comprimento e a largura dos cachos, das bagas e dos engaços, o teor de sólidos solúveis, o pH, a acidez titulável e a relação sólidos solúveis/acidez titulável (SS/AT). O experimento foi conduzido em vinhedo localizado no município de São Miguel Arcanjo, no sudoeste do Estado de São Paulo, sendo os reguladores vegetais aplicados aos 15 dias após o pleno florescimento, mediante pulverização direcionada ao cacho. A interação entre a dose estimada de $5 \mathrm{mg} \mathrm{L}^{-1}$ de forchlorfenuron associada ao $\mathrm{AG}_{3}$ proporcionou os maiores ganhos na massa e na largura das bagas, $e$ as doses estimadas de 4 e $6,5 \mathrm{mg} \mathrm{L}^{-1}$ de forchlorfenuron, associadas ao $A G_{3}$, proporcionaram os menores teores de sólidos solúveis e a relação SS/AT, respectivamente. O tratamento com o uso de $\mathrm{AG}_{3}$ resultou em ganhos no diâmetro dos pedicelos e na massa dos cachos, das bagas e dos engaços, além de incrementar o comprimento e a largura das bagas.

Palavras-chave: Vitis vinifera L., ácido giberélico, forchlorfenuron, qualidade do fruto.

\section{ABSTRACT}

The objective of present research was to evaluate the effects of increasing doses of forchlorfenuron associated, or not, to gibberellic acid on physical and chemical components of the 'Centennial Seedless' grapes. The treatments were gibberellic acid $\left(\mathrm{GA}_{3}\right)$ (0 and $\left.5 \mathrm{mg} \mathrm{L}^{-1}\right)$ associated with forchlorfenuron $\left(0,2,4,6,8\right.$ and $\left.10 \mathrm{mg} \mathrm{L}^{-1}\right)$. The variables were mass, width and length of bunches, berries and rachis, total soluble solids, $\mathrm{pH}$, acidity and total ratio soluble solids/ titratable acidity (SS/TA). The experiment was conducted in a vineyard located in the municipality of São Miguel Arcanjo, southwest of São Paulo State, and the regulators were applied at 15 days after full bloom by spraying the bunch. The interaction of the estimated dose of $5 \mathrm{mg} \mathrm{L}^{-1}$ forchlorfenuron associated with $\mathrm{GA}_{3}$, provided increases on the mass and width of the berries, and the estimated doses of 4 and $6.5 \mathrm{mg} \mathrm{L}^{-1}$ of forchlorfenuron associated with $G_{3}$, showed the lowest soluble solids and ratio SS/TA, respectively. The treatment with $G_{3}$ resulted in gains in the diameter of the pedicels, mass of bunches, berries and rachis, and increasing the length and width of berries.

Key words: Vitis vinifera L., gibberellic acid, forchlorfenuron, fruit quality.

\section{INTRODUÇÃO}

A frutificação natural de videiras sem sementes origina cachos e bagas de dimensões reduzidas. Assim, a utilização de alguns reguladores vegetais para a melhoria das características morfológicas dos cachos e das bagas da videira consiste numa prática cultural imprescindível (PIRES \& BOTELHO, 2001). Segundo POMMER et al. (2003), a cultivar 'Centennial Seedless' produz cachos de tamanhos médios a grandes, levemente soltos, com

IPrograma de Pós-graduação em Agricultura Tropical e Subtropical, Instituto Agronômico (IAC), Campinas, SP, Brasil.

IIAC, CP 28, 13001-920, Campinas, SP, Brasil. E-mail: mmterra@iac.sp.gov.br. *Autor para correspondência.

IIICentro Avançado de Pesquisa Tecnológica do Agronegócio de Frutas, IAC, Jundiaí, SP, Brasil.

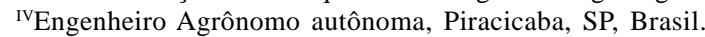


bagas uniformes em tamanho e de baixa aderência ao pedicelo, com grande expressão comercial no Estado de São Paulo.

O uso comercial do ácido giberélico $\left(\mathrm{AG}_{3}\right)$ na viticultura é amplamente difundido, pois visa principalmente a aumentar a fixação e o tamanho das bagas, a descompactação do cacho e a eliminação das sementes (PIRES et al., 2003). O aumento das bagas em uvas apirenas está diretamente relacionado à utilização de reguladores vegetais, em particular ao efeito do $\mathrm{AG}_{3}$ sobre a atividade celular e as mudanças na relação fontedreno dos metabólicos (GOWDA et al., 2006). Porém, PÉREZ \& MORALES (1999) observaram que esse regulador vegetal promove o endurecimento e engrossamento dos pedicelos, pela elevação da atividade de peroxidase solúvel, o que acarretará uma possível degrana dos cachos durante a colheita e especialmente no processo pós-colheita.

Uma opção viável que limita os efeitos indesejáveis do $\mathrm{AG}_{3}$ seria a substituição ou redução deste, com a inserção de outros produtos (PIRES et al., 2003; HAN \& LEE, 2004). O forchlorfenuron (CPPU) é uma citocinina sintética com elevada atividade fisiológica sobre muitas plantas frutíferas, incluindo as videiras, pois também estimula a divisão e expansão celular, além de atrasar a senescência dos tecidos e o amadurecimento do fruto (REYNOLDS et al., 1992; DOKOOZLIAN, 2001).

Em kiwi cultivado na Nova Zelândia, o forchlorfenuron aplicado por imersão e aspersão incrementou o tamanho médio dos frutos em 44 e 33\%, respectivamente, quando comparado à testemunha (PATTERSON et al., 1993). No Canadá, em pesquisa com uvas ‘Sovereign Coronation' e 'Selection 495', o uso de doses crescentes de forchlorfenuron promoveu aumentos lineares na massa fresca dos cachos e das bagas (REYNOLDS et al., 1992).

Este trabalho objetivou avaliar o efeito de doses crescentes do forchlorfenuron associado ou não ao ácido giberélico sobre componentes físico-químicos da uva 'Centennial Seedless' para consumo in natura.

\section{MATERIAL E MÉTODOS}

O experimento foi realizado em vinhedo comercial durante o ano agrícola 2008/09, no município de São Miguel Arcanjo, localizado nas coordenadas geográficas de $23^{\circ} 88^{\prime} \mathrm{S}$ e $47^{\circ} 09^{\prime} \mathrm{O}$, altitude de $660 \mathrm{~m}$, médias anuais de precipitação pluvial de $1.174 \mathrm{~mm}$ e temperatura de $20,4^{\circ} \mathrm{C}$, em solo Argissolo Vermelhoamarelo.

As plantas de 'Centennial Seedless' estavam enxertadas sobre o porta-enxerto ' 420 A', foram conduzidas no sistema latada, no espaçamento de $3,5 \times 3,5 \mathrm{~m}$, e encontravam-se no quinto ano de produção.

Os tratamentos foram aplicados no dia 30 de outubro de 2008 e consistiram nas aplicações de $\mathrm{AG}_{3}$, nas doses de 0 e $5 \mathrm{mg} \mathrm{L}^{-1}$, associados às doses de 0, 2, 4, 6, 8 e 10 $\mathrm{mg} \mathrm{L}^{-1}$ de forchlorfenuron, com adição, na solução aquosa, de $0,3 \mathrm{~mL} \mathrm{~L}^{-1}$ de espalhante adesivo da marca comercial Iharaguen- $S^{\circledR}$. Realizaram-se as aplicações por pulverização direcionada aos cachos, 15 dias após o pleno florescimento, e com diâmetro da baga aproximado de $5 \mathrm{~mm}$. Os demais tratos culturais foram adotados conforme recomendação técnica para a cultura na região.

O delineamento experimental foi em blocos casualizados, em esquema fatorial $2 \times 6$, correspondendo a duas doses de $\mathrm{AG}_{3}$ e seis doses de forchlorfenuron, num total de 12 tratamentos, com cinco repetições cada e dois cachos por parcela.

A colheita dos cachos foi realizada no dia 07 de janeiro de 2009. Em seguida, os cachos foram identificados e levados ao Laboratório do Grupo de Engenharia de Pós-colheita (GEPC), do Instituto de Tecnologia de Alimentos (ITAL), para as avaliações físico-químicas.

As avaliações físicas constaram das seguintes variáveis: massa fresca, comprimento e largura dos cachos, bagas e engaços e diâmetro do pedicelo. Para se obter a massa fresca dos cachos, das bagas e dos engaços, utilizou-se uma balança de precisão digital com $0,1 \mathrm{~g}$ de precisão. Para a determinação do comprimento e da largura dos cachos e engaços, usou-se um paquímetro de madeira, com precisão de $0,1 \mathrm{~cm}$. Para obtenção do comprimento e da largura das bagas, avaliou-se uma subamostra de 10 bagas por cacho, usando uma régua de $30 \mathrm{~cm}$, com precisão de $0,1 \mathrm{~cm}$; e finalmente com um paquímetro digital mediu-se o diâmetro do pedicelo. $\mathrm{O}$ índice de degrana (ID) foi determinado mediante a pesagem do cacho, obtendo-se a porcentagem por meio do seguinte cálculo: $\mathrm{E} \%=[($ massa de bagas soltas $) /($ massa do cacho inteiro) $\mathrm{x}$ 100].

Para as variáveis químicas, avaliaram-se: teor dos sólidos solúveis (SS), com auxílio de refratômetro de bancada (Schmidt Haensch SR400); pH, com leitura direta do suco de 10 bagas, em pHmetro digital (Mettler Toledo modelo 320); e acidez titulável (AT), em pHmetro digital Mettler Toledo, modelo 320, com eletrodo Mettler Toledo, modelo Inlab 413, por titulação em uma alíquota de $5 \mathrm{ml}$ do mosto, com $\mathrm{NaOH}$ 0,1N, e expressa em g de ácido tartárico por 100ml de mosto (CARVALHO et al., 1990). 
As médias foram submetidas à análise de variância e ao teste de Tukey, com 5\% de significância, para comparar o efeito das doses do $\mathrm{AG}_{3}$, à análise de regressão polinomial, para as doses de forchlorfenuron.

\section{RESULTADOS E DISCUSSÃO}

Verificou-se interação significativa entre as doses de GA e forchlorfenuron para as variáveis diâmetro do pedicelo; massa e largura das bagas; massa do engaço (Tabela 1); e teor de sólidos solúveis e relação (SS/AT) (Tabela 2). Para as variáveis massa e largura das bagas, o uso do forchlorfenuron associado ao $\mathrm{AG}_{3}$ apresentou significância quadrática, sendo a dose estimada de $5 \mathrm{mg} \mathrm{L}^{-1}$ de forchlorfenuron $+5 \mathrm{mg} \mathrm{L}^{-1}$ de $\mathrm{AG}_{3}$ como aquela que proporcionou maiores médias de massa (Figura 1a) e largura (Figura 1b) das bagas, com incrementos de 13 e 2,5\%, respectivamente. Esse resultado é similar ao encontrado por HAN \& LEE (2004) na Coreia do Sul, com videira 'Kyoho', que obtiveram ganhos na massa das bagas e dos cachos, com a utilização de $10 \mathrm{mg} \mathrm{L}^{-1}$ de forchlorfenuron, associado a $25 \mathrm{mg} \mathrm{L}^{-1}$ do $\mathrm{AG}_{3}$, aplicado 10 dias após o pleno florescimento. Eessas respostas corroboram a pesquisa de GOWDA et al. (2006), os quais afirmam que, em razão do efeito dos reguladores vegetais, as bagas de uva expandem-se pelo aumento da atividade de divisão celular em fase inicial do desenvolvimento e pelo influxo de água e transporte de metabólicos na fase final do desenvolvimento dos frutos.

Para diâmetro do pedicelo (Figura 1c) e massa do engaço (Figura 1d), a aplicação de doses crescentes de forchlorfenuron, associado ao $\mathrm{AG}_{3}$, resultou em ganhos lineares. Esse efeito é semelhante ao encontrado por PÉREZ \& MORALES (1999), os quais, com a utilização de doses crescentes de $A G_{3}$, observaram aumento da atividade da enzima peroxidase e este correlacionado com o ganho na massa do pedicelo.

Na composição química dos frutos, as doses estimadas de 4 e $6,5 \mathrm{mg} \mathrm{L}^{-1}$ de forchlorfenuron com $\mathrm{AG}_{3}$ foram aquelas que apresentaram menores valores na concentração de sólidos solúveis (SS) e da relação SS/ AT, respectivamente; já o uso do forchlorfenuron sem o $\mathrm{AG}_{3}$ não apresentou efeito significativo sobre essas variáveis (Figura 2). Resultados análogos foram observados por RIBEIRO \& SCARPARE FILHO (2003), que detectaram redução do SS quando adicionado o forchlorfenuron a doses de $\mathrm{AG}_{3}$.

Para as demais variáveis avaliadas, não se verificou interação significativa entre o forchlorfenuron e o $A_{3}$, havendo, no entanto, efeito isolado dos tratamentos.

Na utilização de doses crescentes de forchlorfenuron, sem $\mathrm{AG}_{3}$, observaram-se aumentos lineares para massa (Figura 3a) e comprimento (Figura 3b) dos cachos e no comprimento dos engaços, (Figura 3c). Esse aumento nas massas dos cachos e das bagas, com o uso do CPPU, também foi reportado por outros autores em diversas cultivares de uvas (REYNOLDS et al., 1992; PIRES et al., 2003; TECCHIO et al., 2006). Neste trabalho, observou-se que o aumento na massa do engaço (Figura 1d), promovido pelo CPPU associado ou não ao $\mathrm{AG}_{3}$, relacionou-se com o incremento da massa do cacho (Figura 3a).

Tabela 1 - Quadrado médio, média e coeficiente de variação das variáveis: diâmetro do pedicelo (DP); índice de degrana (ID); massa, comprimento e largura dos cachos, das bagas e dos engaços da uva 'Centennial Seedless', São Miguel Arcanjo, SP, 2009.

\begin{tabular}{|c|c|c|c|c|c|c|c|c|c|c|c|c|}
\hline \multirow[b]{2}{*}{$\begin{array}{l}\text { Fator de } \\
\text { Variação }\end{array}$} & \multirow[b]{2}{*}{ GL } & \multirow[b]{2}{*}{$\begin{array}{c}\mathrm{DP} \\
(\mathrm{mm})\end{array}$} & \multirow[b]{2}{*}{ ID (\%) } & \multicolumn{3}{|c|}{------------'Cacho------------- } & \multicolumn{3}{|c|}{-------------Baga------------- } & \multicolumn{3}{|c|}{------------Engaço------------- } \\
\hline & & & & $\begin{array}{l}\text { Massa } \\
\text { (g) }\end{array}$ & $\begin{array}{l}\text { Comp. } \\
\text { (cm) }\end{array}$ & $\begin{array}{l}\text { Larg. } \\
(\mathrm{cm})\end{array}$ & $\begin{array}{l}\text { Massa } \\
\text { (g) }\end{array}$ & $\begin{array}{l}\text { Comp. } \\
\text { (cm) }\end{array}$ & $\begin{array}{l}\text { Larg. } \\
\text { (cm) }\end{array}$ & $\begin{array}{l}\text { Massa } \\
\text { (g) }\end{array}$ & $\begin{array}{l}\text { Comp. } \\
\text { (cm) }\end{array}$ & $\begin{array}{l}\text { Larg. } \\
\text { (cm) }\end{array}$ \\
\hline Blocos & 4 & $0,054^{\text {ns }}$ & $0,580^{\mathrm{ns}}$ & $19235^{\text {ns }}$ & $5,307^{\text {ns }}$ & $5,213^{\mathrm{ns}}$ & $1,023^{\mathrm{ns}}$ & $0,096^{\mathrm{ns}}$ & $0,001^{\mathrm{ns}}$ & $1,540^{\text {ns }}$ & $4,154^{\mathrm{ns}}$ & $1,643^{\mathrm{ns}}$ \\
\hline $\mathrm{AG}_{3}$ & 1 & $5,412^{*}$ & $4,947^{\mathrm{ns}}$ & 278619* & $10,127^{*}$ & $0,047^{\mathrm{ns}}$ & $28,483^{*}$ & $1,626^{*}$ & $0,183^{*}$ & $198,67^{*}$ & $0,726^{\mathrm{ns}}$ & $0,002^{\mathrm{ns}}$ \\
\hline CPPU & 5 & $2,738^{*}$ & $2,050^{\mathrm{ns}}$ & 234395* & $6,979^{*}$ & $1,951^{\mathrm{ns}}$ & $3,923^{*}$ & $0,189 *$ & $0,034^{*}$ & $292,92 *$ & $12,50 *$ & $1,955^{\mathrm{ns}}$ \\
\hline $\begin{array}{l}\text { Interação } \\
\mathrm{AG}_{3} \quad \mathrm{x} \\
\mathrm{CPPU}\end{array}$ & 5 & $0,370^{*}$ & $2,854^{\mathrm{ns}}$ & $39676^{\mathrm{ns}}$ & $2,364^{\text {ns }}$ & $1,617^{\text {ns }}$ & $2,579 *$ & $0,198^{\text {ns }}$ & $0,024^{*}$ & $33,87^{*}$ & $0,413^{\text {ns }}$ & $2,079^{\mathrm{ns}}$ \\
\hline Resíduo & 44 & 0,108 & 2,236 & 19805 & 1,923 & 0,929 & 0,629 & 0,050 & 0,004 & 12,39 & 3,526 & 0,987 \\
\hline CV (\%) & - & 7,67 & 124,79 & 17,04 & 6,53 & 6,55 & 10,70 & 7,17 & 3,18 & 19,96 & 9,59 & 10,93 \\
\hline Média & - & 4,30 & 1,19 & 825,92 & 21,25 & 14,65 & 7,41 & 3,12 & 2,03 & 17,64 & 19,58 & 9,09 \\
\hline
\end{tabular}

${ }^{\text {ns }}$ não significativo, * Teste F significativo $(\mathrm{P} \leq 0,05)$. 
Tabela 2 - Quadrado médio, média e coeficiente de variação das variáveis: sólidos solúveis (SS), pH, acidez titulável (AT) e relação SS/AT da uva ‘Centennial Seedless’, São Miguel Arcanjo, SP, 2009.

\begin{tabular}{llllll}
\hline Fator de Variação & GL & SS ( ${ }^{\circ}$ Brix) & pH & AT (g ácido tartárico 100ml ${ }^{-1}$ de suco) & SS/AT \\
\hline Blocos & 4 & $0,28^{\text {ns }}$ & $0,001^{\text {ns }}$ & $0,005^{\text {ns }}$ & $0,706^{\text {ns }}$ \\
$\mathrm{AG}_{3}$ & 1 & $32,017^{*}$ & $0,185^{*}$ & $014^{*}$ & $0,002^{\text {ns }}$ \\
CPPU & 5 & $10,058^{*}$ & $0,009^{*}$ & $0,003^{\text {ns }}$ \\
$\mathrm{AG}_{3}$ X CPPU & 5 & $5,245^{*}$ & $0,006^{\text {ns }}$ & 0,002 & $24,077^{*}$ \\
Resíduo & 44 & 0,811 & 0,003 & 0,63 & 5,959 \\
Média & - & 14,74 & 3,23 & 8,26 \\
CV $(\%)$ & - & 6,11 & 1,77 & 23,69 \\
\hline
\end{tabular}

${ }^{\text {ns }}$ não significativo, * Teste F significativo $(\mathrm{P} \leq 0,05)$.

O uso de doses crescentes de forchlorfenuron apresentou efeito quadrático sobre o comprimento da baga, com a dose estimada de $3 \mathrm{mg} \mathrm{L}^{-1}$ desse regulador vegetal, proporcionando o menor comprimento das bagas (Figura 3d). Embora tenha apresentado regressão significativa, o coeficiente de determinação dessa variável foi baixo $\left(R^{2}=0,43\right)$, resultado divergente do observado por RIBEIRO \& SCARPARE FILHO (2003), os quais, em uva 'Centennial Seedless', obtiveram maiores comprimentos das bagas na interação entre o $\mathrm{AG}_{3}$ e o forchlorfenuron, em comparação com o uso isolado do $\mathrm{AG}_{3}$.
De acordo com a tabela 3, nos tratamentos em que foram aplicados $5 \mathrm{mg} \mathrm{L}^{-1}$ do $\mathrm{AG}_{3}$, isoladamente, foram observadas respostas significativas com ganhos nas variáveis: massa dos cachos e comprimento das bagas, além de redução no comprimento dos cachos. Já para as variáveis químicas obteve-se o aumento na acidez titulável (AT), e esse regulador vegetal também levou a uma redução no $\mathrm{pH}$ dos frutos, sugerindo que seu uso nessa cultivar favoreça o atraso na maturação destes, prorrogando o período da colheita, pois, segundo Instrução normativa $\mathrm{N}^{\circ} 001$ de fevereiro de 2002, os frutos de uva deverão apresentar SS superior a $14^{\circ}$ Brix, para comercialização (BRASIL, 2002).

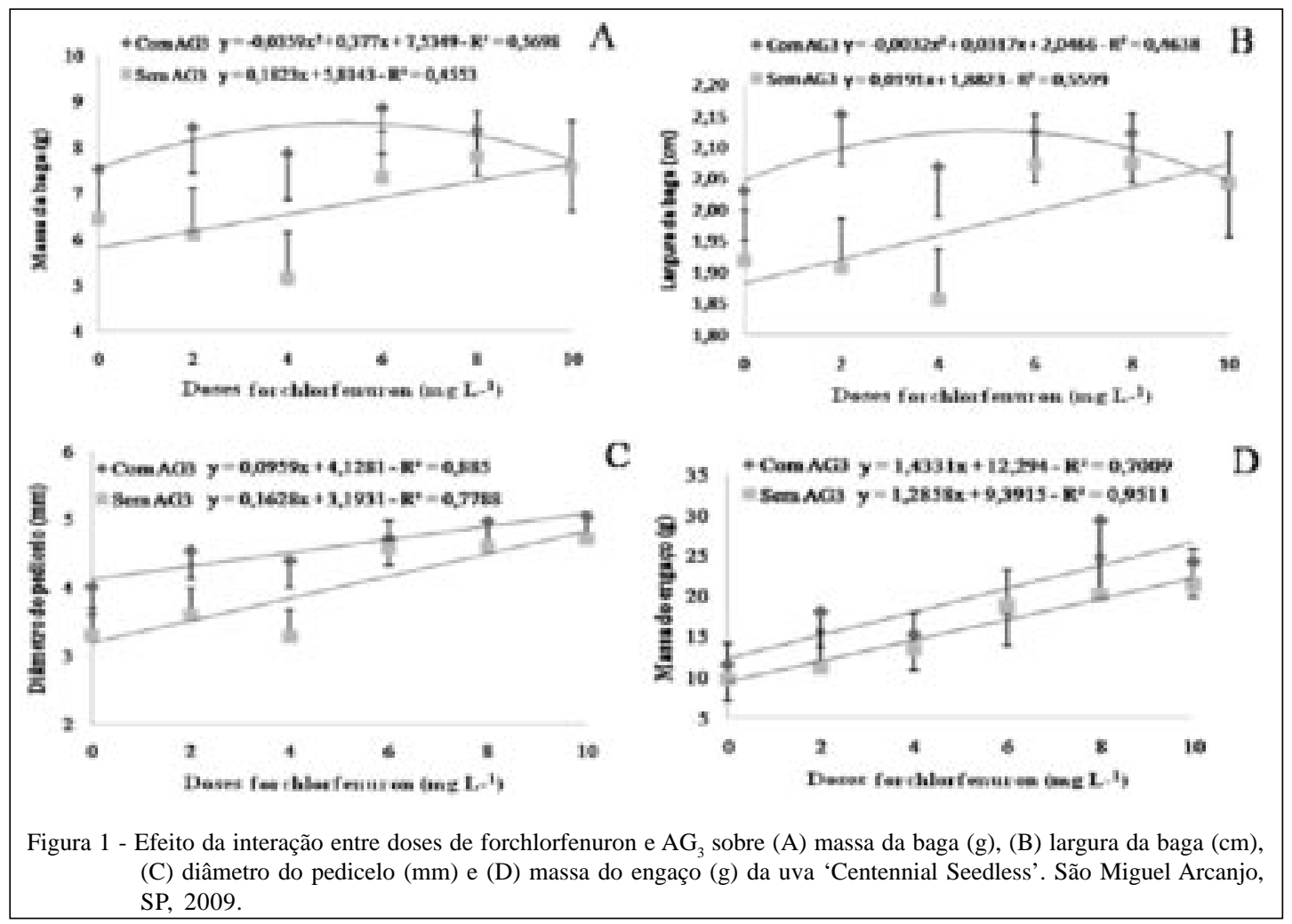

Ciência Rural, v.40, n.8, ago, 2010. 


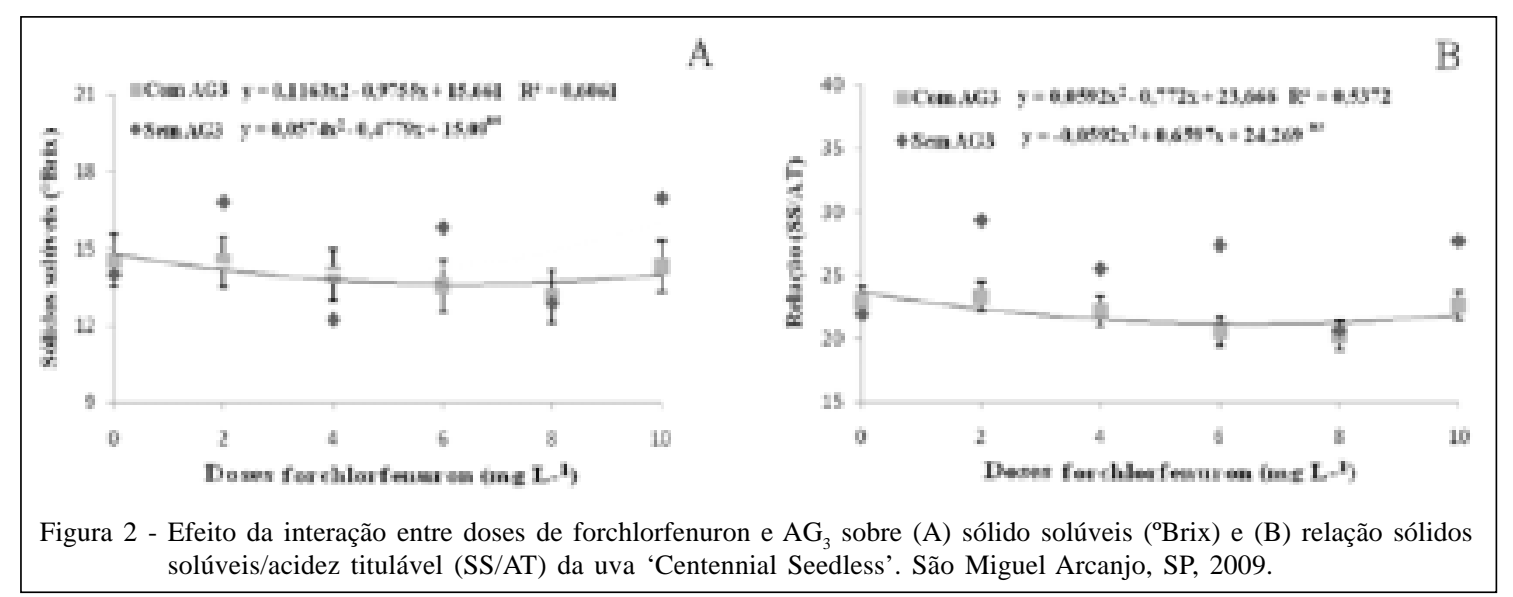

GOWDA et al. (2006), na Índia, obtiveram resultados semelhantes com a cultivar 'Thompson Seedless', em que a aplicação de doses crescentes de $\mathrm{AG}_{3}$, durante o desenvolvimento da planta, resultou em ganhos na massa, no comprimento e na largura dos cachos e das bagas, mas não observaram diferenças significativas sobre os SS e a AT.

\section{CONCLUSÃO}

A dose estimada de $5 \mathrm{mg} \mathrm{L}^{-1}$ do forchlorfenuron, associada à dose de $5 \mathrm{mg} \mathrm{L}^{-1}$ do $\mathrm{AG}_{3}$, aumentou significativamente a massa e largura das bagas, o que demonstra sua elevada capacidade para uso comercial, de modo que a aplicação do forchlorfenuron e $\mathrm{AG}_{3}$ não prejudicou o processo póscolheita, ou seja, não aumentou o índice de degrana (ID) dos cachos. Entretanto, esses reguladores vegetais atrasaram a maturação dos frutos, devido à redução dos valores de SS e da relação SS/AT.

\section{AGRADECIMENTOS}

À Nilce Ribeiro Vaz de Sales, por permitir a realização do experimento em sua propriedade, e aos funcionários do laboratório GEPC do ITAL, pelo auxílio nas análises físico-químicas.

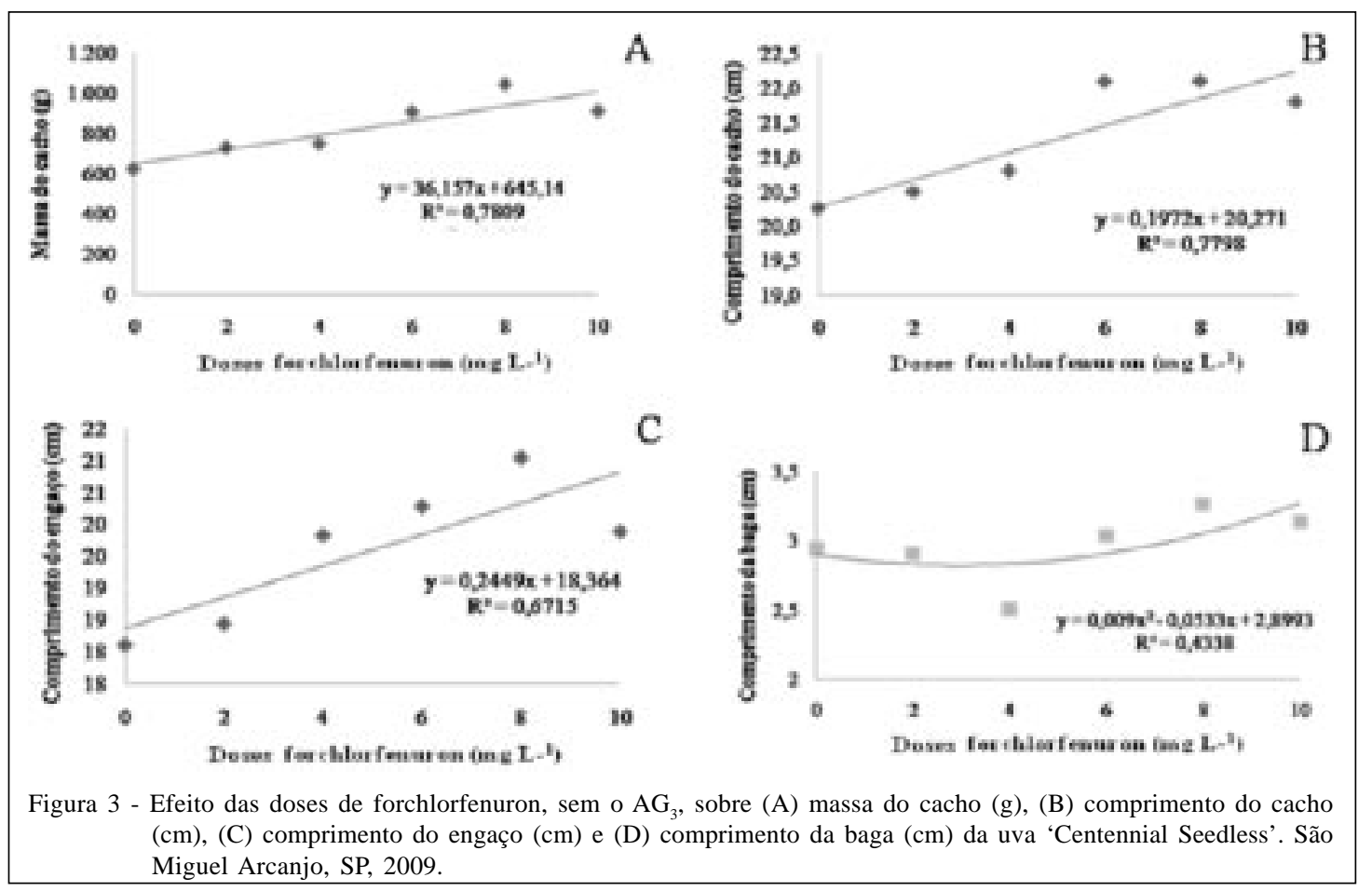

Ciência Rural, v.40, n.8, ago, 2010. 
Tabela 3 - Efeito de doses crescentes do forchlorfenuron ou do AG $_{3}$ sobre variáveis físico-químicas da uva 'Centennial Seedless', São Miguel Arcanjo, SP, 2009.

\begin{tabular}{|c|c|c|c|c|c|c|c|c|c|}
\hline \multirow{2}{*}{$\begin{array}{c}\text { Fator de } \\
\text { Variação } \\
\\
\mathrm{AG}_{3} \\
\left(\mathrm{mg} \mathrm{L}^{-1}\right)\end{array}$} & \multirow{2}{*}{ ID (\%) } & \multicolumn{3}{|c|}{-----------------Cacho----------------- } & \multirow{2}{*}{$\begin{array}{l}\text { Baga } \\
\text { Comp. } \\
\text { (cm) }\end{array}$} & \multicolumn{2}{|c|}{--------Engaço--------- } & \multirow{2}{*}{$\mathrm{pH}$} & \multirow{2}{*}{$\begin{array}{c}\text { AT } \\
\text { (g ácido tartárico } \\
100 \mathrm{ml}^{-1} \text { de suco) }\end{array}$} \\
\hline & & Massa (g) & $\begin{array}{l}\text { Comp. } \\
\text { (cm) }\end{array}$ & $\begin{array}{l}\text { Largura } \\
\text { (cm) }\end{array}$ & & $\begin{array}{l}\text { Comp. } \\
\text { (cm) }\end{array}$ & $\begin{array}{l}\text { Largura } \\
\text { (cm) }\end{array}$ & & \\
\hline 0 & $1,48 \mathrm{a}$ & $757,78 \mathrm{~b}$ & $21,66 \mathrm{a}$ & $14,62 \mathrm{a}$ & $2,96 \mathrm{~b}$ & $19,47 \mathrm{a}$ & 9,08 a & $3,28 \mathrm{a}$ & $0,61 \mathrm{~b}$ \\
\hline 5 & $0,91 \mathrm{a}$ & 894,07 a & $20,84 \mathrm{~b}$ & $14,65 \mathrm{a}$ & 3,29 a & 19,69 a & 9,09 a & $3,17 \mathrm{~b}$ & $0,64 \mathrm{a}$ \\
\hline $\begin{array}{l}\text { DMS } \\
\text { (Tukey) }\end{array}$ & 0,77 & 73,23 & 0,72 & 0,49 & 0,11 & 0,97 & 0,51 & 0,02 & 0,02 \\
\hline Teste F & $2,12^{\mathrm{ns}}$ & $14,06 *$ & $5,26 *$ & $0,05^{\mathrm{ns}}$ & $32,30 *$ & $0,20^{\mathrm{ns}}$ & $0,00^{\mathrm{ns}}$ & $56,69 *$ & $5,37 *$ \\
\hline \multicolumn{10}{|l|}{$\begin{array}{l}\text { CPPU } \\
\left(\mathrm{mg} \mathrm{L}^{-1}\right)\end{array}$} \\
\hline 0 & 1,97 & 622,00 & 20,25 & 14,88 & 3,07 & 18,10 & 9,00 & 3,21 & 0,64 \\
\hline 2 & 0,86 & 730,16 & 20,50 & 14,17 & 3,13 & 18,43 & 8,54 & 3,27 & 0,60 \\
\hline 4 & 0,96 & 746,76 & 20,80 & 14,19 & 2,89 & 19,82 & 8,79 & 3,20 & 0,64 \\
\hline 6 & 0,89 & 904,77 & 22,09 & 14,95 & 3,23 & 20,27 & 9,43 & 3,26 & 0,63 \\
\hline 8 & 1,52 & 1042,8 & 22,20 & 15,23 & 3,28 & 21,03 & 9,77 & 3,22 & 0,64 \\
\hline 10 & 0,96 & 908,98 & 21,79 & 14,47 & 3,13 & 19,87 & 9,01 & 3,20 & 0,63 \\
\hline regressão & Ns & $\mathrm{L} *$ & $\mathrm{~L}^{*}$ & ns & $\mathrm{Q}^{*}$ & $\mathrm{~L}^{*}$ & ns & ns & Ns \\
\hline \multicolumn{10}{|l|}{$\begin{array}{l}\text { Interação } \\
\mathrm{AG}_{3} \text { x CPPU }\end{array}$} \\
\hline Teste F & $1,27^{\text {ns }}$ & 2,00 ns & $1,22^{\mathrm{ns}}$ & $1,75^{\text {ns }}$ & $3,94^{\mathrm{ns}}$ & $0,11^{\text {ns }}$ & $2,10^{\mathrm{ns}}$ & $1,86^{\mathrm{ns}}$ & $1,21^{\mathrm{ns}}$ \\
\hline CV\% & 124,79 & 17,04 & 6,53 & 6,55 & 7,17 & 9,59 & 10,93 & 1,77 & 8,26 \\
\hline
\end{tabular}

Médias com letras iguais, na coluna, não diferem entre si para o teste de Tukey, a 5\%; ns: não significativo; * Teste $\mathrm{F}$ significativo (P $\leq 0,05$ ); L: regressão linear; Q: regressão quadrática.

\section{REFERÊNCIAS}

BRASIL. Ministério da Agricultura. Instrução Normativa de 01 de fevereiro de 2002. Anexo II. Regulamento Técnico de Classificação da Uva Fina de Mesa. Disponível em: <http:// www.pr.gov.br/claspar/pdf/uvarustica.pdf>. Acesso em: 15 nov. 2009.

CARVALHO, C.R.L. et al. Análises químicas de alimentos. Campinas: ITAL, 1990. 121p.

DOKOOZLIAN, N.K. CPPU: A potential new plant growth regulator for California table grapes. Grape Notes. Oakland: UCCE, 2001. 4p. Disponível em: <http://cetulare.ucdavis.edu/ pub/gra0301.pdf>. Acesso em: 10 ago. 2009.

GOWDA, V.N. et al. Influence of $\mathrm{GA}_{3}$ on growth and development of 'Thompson Seedless' grapes (Vitis vinifera L.). Acta Horticulturae, Leuven, n.727, p.239-242, 2006.

HAN, D.H.; LEE, C.H. The effects of $\mathrm{GA}_{3}$, CPPU and ABA applications on the quality of kyoho (Vitis vinifera $\mathrm{L} . \mathrm{X} \mathbf{V}$. labrusca L.) grape. Acta Horticulturae, Leuven, n.653, p.193-197, 2004.

PATTERSON, K.J. et al. Effects of CPPU (N-(2-chloro-4pyridyl)-N'-phenylurea) on fruit growth, maturity, and storage quality of kiwifruit. New Zealand Journal of Crop and Horticultural Science. Wellington. v.21, p.253-261, 1993.

PÉREZ, F.J.; MORALES, V. A basic peroxidase isoenzyme from the grape pedicel is induced by gibberelic acid. Australian Journal Plant Physiology, Collingwood, v.26, p.387-390, 1999.
PIRES, E.J.P. et al. Efeitos do CPPU e do ácido giberélico nas características dos cachos da uva de mesa 'Centennial Seedless'. Ciência e Agrotecnologia, Lavras, v.27, n.2, p.305-311, 2003.

PIRES, E.J.P.; BOTELHO, R.V. Uso de reguladores vegetais na cultura da videira. In: SIMPÓSIO BRASILEIRO DE UVAS DE MESA, 2000, Ilha Solteira. Anais... Ilha Solteira: [s. n.], 2001. p.129-147.

POMMER, C.V. et al. Cultivares de videira. In: POMMER, C.V. (Ed). Uva: tecnologia de produção, pós-colheita, mercado. Porto Alegre: Cinco Continentes, 2003. p.109249.

REYNOLDS, A.G. et al. Phenylureas CPPU and Thiadizuron affect yield components, fruit composition, and storage potential of four seedless grape selections. Journal of the American Society for Horticultural Science, New York, v.117, n.1, p.85-89, 1992.

RIBEIRO, V.G.; SCARPARE FILHO, J.A. Crescimento de bagas de cultivares de uvas apirênicas tratadas com CPPU e GA . Ciência e Agrotecnologia, Lavras, v. 27, n.6, p.305-311, 2003.

TECCHIO, M.A. et al. Efeitos do CPPU e do ácido giberélico nas características morfológicas dos cachos e bagos da uva 'Vênus'. Acta Scientiarum Agronomy. Maringá, v.28, n.4, p.507-511, 2006. Disponível em: <http://periodicos.uem.br/ ojs/index.php/ ActaSciAgron/article/view/784/456>. Acesso em: 18 out. 2009. doi: 10.4025/actasciagron. v28i4.784. 